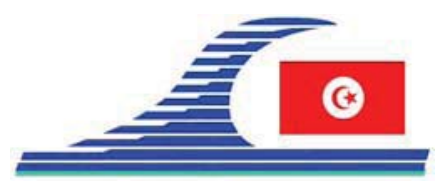

Conférence Méditerranéenne Côtière et Maritime

EDITION 1, HAMMAMET, TUNISIE (2009)

Coastal and Maritime Mediterranean Conference

Disponible en ligne - http://www.paralia.fr-Available online

\title{
Applications de filtres géotextiles à la protection côtière
}

\section{Aristide HEHNER ${ }^{1}$, Georg HEERTEN ${ }^{2}$}

1. Naue Applications, Vienne, France.

naueapplications@wanadoo.fr

2. NAUE GmbH \& Co. KG, Management, Espelkamp-Fiestel, Germany.

\section{Résumé :}

L'ingénierie hydraulique fut le domaine de départ de la conception des géotextiles. Pour des raisons économiques, techniques et leurs avantages écologiques, les filtres géotextiles sont ainsi utilisés pour les fonctions de filtration et de drainage, et ceci en constante augmentation dans le monde entier depuis plus de 40 ans.

Les produits employés doivent être conçus et sélectionnés en fonction de l'application et des exigences spécifiques de chaque ouvrage, mais doivent également être installés sans dommage. Une capacité d'allongement élevée confère au filtre géotextile sa résistance lors des sollicitations en période de construction, phase qui présente le plus important risque d'endommagement dans la vie du géotextile. Le document mettra l'accent sur des exemples d'application des géotextiles non-tissés aiguilletés dans le domaine de la construction hydraulique et de la protection côtière.

\section{Mots clefs :}

Génie côtier - Hydraulique maritime - Géoconteneur - Filtre géotextile Environnement littoral - Protection côtière - Erosion

\section{Principes de base}

Un filtre utilisé dans la géotechnique et dans le génie hydraulique doit satisfaire la quasi-contradiction de la rétention et de la perméabilité. Encore aujourd'hui, certains concepteurs supposent à tort que ces deux critères sont les seuls nécessaires pour le bon fonctionnement d'un filtre géotextile. Il est important de souligner que l'épaisseur, la masse surfacique et la capacité d'allongement à la rupture sont de très importantes exigences pour la conception et l'installation de filtres géotextiles. La vérification des paramètres d'ouverture de filtration et de robustesse à la perforation sont des étapes indispensables dans la conception d'un ouvrage de protection côtière.

\section{Etat de l'art}

\subsection{Conception et spécifications}

Dans la pratique actuelle, la conception de filtre géotextile se limite à la détermination de l'ouverture de filtration et de la perméabilité voire la permittivité. Les exigences au DOI: $10.5150 / \mathrm{cmcm} .2009 .051-0$ 
niveau de l'épaisseur sont encore plutôt rares, de même les spécifications sur les critères pour éviter la perforation lors de la mise en œuvre.

On compare les dimensions d'un filtre généralement représentées par l'ouverture de filtration caractéristique du géotextile suivant la norme EN ISO 12956 Géotextiles et produits apparentés - détermination de l'ouverture de filtration-, à la dimension d85 des grains formant le sol. La dimension d85 est obtenue sur la courbe granulométrique, et correspond à la maille du tamis qui laisse passer $85 \%$ du poids du sol. Il s'agit donc des plus gros grains du sol en question.

L'ouverture de filtration O90 est habituellement requise pour être plus petite que dy (paramètre définissant la courbe granulométrique du sol) multiplié par le facteur $\mathrm{x}$.

O90 $<\mathrm{x}$ dy

Ce critère de conception ne connaît pas de limite inférieure pour les plus petites ouvertures afin d'éviter le phénomène de colmatage. En conséquence, le filtre géotextile peut se colmater avec une diminution de sa perméabilité (HEERTEN, 1993). Le simple critère que la perméabilité du filtre géotextile devrait être de 10 à 100 fois plus élevée que la perméabilité du sol, ne satisfait pas une bonne conception d'un filtre géotextile. Par conséquent, la conception et la spécification des critères pour les filtres géotextiles doivent satisfaire les critères suivants :

- Une limite inférieure pour l'ouverture de filtration;

- Épaisseur / longueur du chemin de filtration (parcours);

- Critères de sécurité à l'installation afin d'éviter l'endommagement mécanique du géotextile.

\subsection{Analogies filtre granulaire / filtre géotextile}

L'analogie tient compte d'une part de la taille et du volume des ouvertures, d'autre part de l'épaisseur. La comparaison est uniquement valable pour des géotextiles filtres aiguilletés à base de fibres coupées car seul ce procédé de fabrication permet d'obtenir un espace tridimensionnel tel qu'il est formé par le filtre granulaire.

Le principe de base de cette analogie consiste en fait à l'idée d'offrir à un filtre géotextile "la même" structure d'ouverture que celle formée par un matériau granulaire, partant du fait que les éléments de filtre sont les ouvertures et non pas les éléments (grains/fibres) formant les ouvertures. Cette analogie a été étudiée par PRAPAHARAN et al. (1989) sur des géotextiles non-tissés à l'aide de la méthode intrusion de mercure indiquant la taille et la distribution des ouvertures d'environ $0,1 \mathrm{~mm}$ à $0,2 \mathrm{~mm}$. Ce spectre d'ouverture fait référence à une ouverture type d'un sable moyen (diamètre $\mathrm{d}=0,2$ à $0,63 \mathrm{~mm})$.

\subsection{Dimensionnement de filtres géotextiles}

Avec l'analogie par rapport aux matériaux granulaires concernant l'ouverture de 
filtration et l'épaisseur, la conception de filtre géotextile est possible : Avec d50 et la granulométrie du sol, une ouverture de filtration O90 d'un filtre approprié peut être déterminée :

O90,d $<$ O90 <0,8 O90,d

Où :

O90, $\mathrm{d}=$ ouverture de filtration "déterminée"

d50 est obtenu sur la courbe granulométrique, et correspond à la maille du tamis qui laisse passer $50 \%$ du poids du sol.

L'épaisseur e du filtre géotextile (e,gtx) doit être limitée par :

25 O90,d $<$ e,gtx $<50$ O90,d

où :

O90,d = ouverture de filtration "déterminée",

e,gtx = épaisseur du géotextile

Avec ces exigences concernant l'ouverture de filtration et l'épaisseur des géotextiles non-tissés, une perméabilité à long terme du filtre géotextile peut être évaluée.

\subsection{Critères de survie pour éviter la perforation du filtre géotextile}

$\mathrm{Au}$ cours de son installation, le filtre géotextile est exposé à différents risques d'endommagementLivré au risque de perforation, tout dimensionnement est inutile. Les sources de danger peuvent varier depuis les manipulations, lors du remplissage et le transport, le remplissage et compactage d'une tranchée, d'un revêtement brise-lames ou d'ouvrages divers dans le cadre de la construction. Seuls les géotextiles non-tissés aiguilletés ont une capacité de déformation élevée (généralement $>40 \%$ ) et avec une masse surfacique $>300 \mathrm{~g} / \mathrm{m}^{2}$ lui conférant une grande résistance à la perforation et à l'abrasion comme il est démontré par des essais d'impact, développés par l'Office National Allemand des Voies Navigables (BAW), dans les années 1970 et expliqués plus en détail par HEERTEN (2005).

\section{Exemple}

Des géoconteneurs fabriqués à partir de filtres géotextiles présentent une solution fiable et économique. Le sable, disponible sur place peut être utilisé pour le remplissage des géoconteneurs qui seront ensuite disposés de manière prédéfinie. La dune ainsi renforcée absorbe l'énergie des différents impacts dus aux vagues et à la houle lesquels sont dissipés par chaque fibre du géotextile. Afin d'empêcher les agressions mécaniques et toute possible influence environnementale, il est recommandé de recouvrir l'installation par du sable et si possible revégétaliser la zone concernée. 


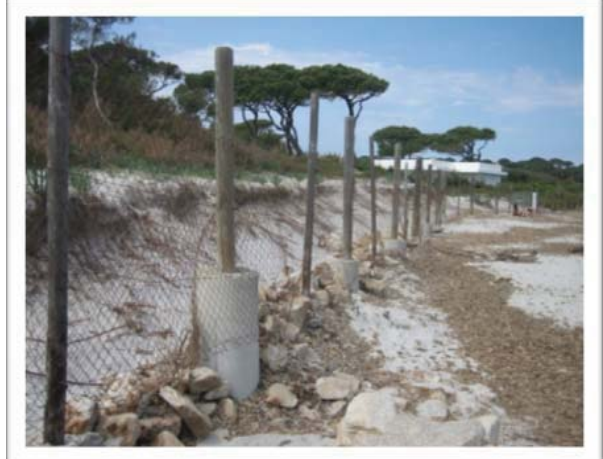

Figure 1. Erosion du massif dunaire sur la côte varoise (France).

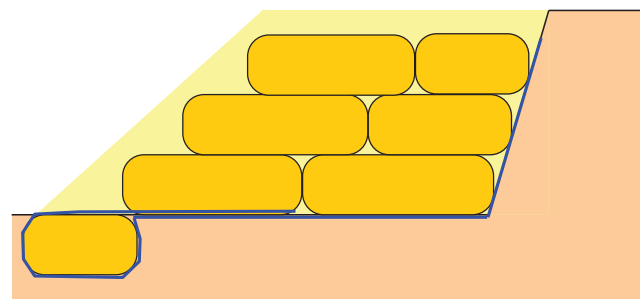

Figure 2. Coupe schématique d'une construction Soft Rock avec des géoconteneurs Terrafix, type E, côte varoise (France).

\section{Conclusion}

En raison des avantages en termes de coût et de la facilité d'installation, les filtres géotextiles et les géosynthétiques de drainage sont de plus en plus utilisés et de nombreux cas illustrent leur fiabilité. L'analogie entre un filtre minéral et un filtre géotextile se base sur le simple fait que ce sont les ouvertures qui constituent l'élément de filtration (et non les particules formant les ouvertures). Cette analogie confère aux concepteurs une sécurité de son dimensionnement. En dehors des "critères de filtres " la robustesse et la durabilité sont des critères indispensable afin d'éviter tout type d'endommagement lors de la mise en œuvre et lors de sollicitations après achèvement. Elle se traduit par la spécification d'une épaisseur minimale, d'une résistance à la traction et d'une résistance à la perforation. L'utilisation de filtres géotextiles non tissés en géoconteneurs démontre clairement l'avantage au niveau de ses caractéristiques à l'allongement. Comme la résistance à la traction est plus ou moins pertinente dans des structures souples et la rupture à la traction est souvent à l'origine de défaillances et de sinistres, le concepteur, selon l'avis des auteurs, devrait raisonner plus en terme d'allongement qu'en terme de résistance à la traction.

\section{Références bibliographiques}

HEERTEN G. (1993). Stand der untersuchung und bemessung des filterverhaltens von Geokunststoff-Boden-Systemen. 3. Informations- und Vortragsveranstaltung über "Kunststoffe in in der Geotechnik", Technische Universität München.

HEERTEN G., WERTH K. (2005). Use of geosynthetics in hydraulic engineering. Baltic Geotechnics X, Riga, Latvia.

PRAPAHARAN S., HOLTZ R.D., LUNA J.D. (1989). Pore size distribution of nonwoven geotextiles. Geotechnical Testing Journal, GTJODJ, Vol. 12, No. 4, pp 261268. 